\title{
Power allocation over time-varying multiple-access interference channels
}

DOI:

10.1002/dac.3158

\section{Document Version}

Accepted author manuscript

Link to publication record in Manchester Research Explorer

\section{Citation for published version (APA):}

Baidas, M. W., Alsusa, E., \& Hamdi, K. (2016). Power allocation over time-varying multiple-access interference channels. International Journal of Communication Systems. https://doi.org/10.1002/dac.3158

\section{Published in:}

International Journal of Communication Systems

\section{Citing this paper}

Please note that where the full-text provided on Manchester Research Explorer is the Author Accepted Manuscript or Proof version this may differ from the final Published version. If citing, it is advised that you check and use the publisher's definitive version.

\section{General rights}

Copyright and moral rights for the publications made accessible in the Research Explorer are retained by the authors and/or other copyright owners and it is a condition of accessing publications that users recognise and abide by the legal requirements associated with these rights.

\section{Takedown policy}

If you believe that this document breaches copyright please refer to the University of Manchester's Takedown Procedures [http://man.ac.uk/04Y6Bo] or contact uml.scholarlycommunications@manchester.ac.uk providing relevant details, so we can investigate your claim.

\section{OPEN ACCESS}




\title{
Power Allocation Over Time-Varying Multi-User Multi-Relay Amplify-and-Forward Networks
}

\author{
Mohammed W. Baidas $\dagger$, Emad Alsusa $\ddagger$, and Khairi A. Hamdi $\ddagger$ \\ $\dagger$ Department of Electrical Engineering, College of Engineering and Petroleum, Kuwait University, Kuwait \\ $\ddagger$ School of Electrical and Electronic Engineering, University of Manchester, Manchester, United Kingdom \\ (email: Baidas@ieee.org, E.Alsusa@manchester.ac.uk, and K.Hamdi@manchester.ac.uk)
}

\begin{abstract}
In this paper, power allocation over time-varying multi-user multi-relay amplify-and-forward networks is studied. Specifically, stochastic network sum-rate, max-min rate power allocation and total power minimization problems are formulated. However, solving such stochastic problems relies on perfect global instantaneous channel state information (CSI), and thus entails complex computations and excessive communication overheads. To circumvent these issues, second-order statistics of the CSI (i.e. partial CSI) are utilized to transform the stochastic formulations into deterministic optimization problems in terms of ergodic capacity while satisfying quality-of-service constraints via target outage probability. The obtained optimal deterministic problems are non-convex and thus are computationally prohibitive. However, at high enough signal-to-noise ratio, such problems can be transformed into asymptotically convex ones, and thus solved efficiently. Simulation results illustrate that the proposed approximate deterministic power allocation reformulations closely agree with their optimal exact deterministic and dynamic counterparts.
\end{abstract}

\section{Index Terms}

Max-min, network sum-rate, outage probability, power allocation, relay channels, quality-of-service

\section{INTRODUCTION}

Cooperative relay networks have been proposed to mimic multiple-input multiple-output (MIMO) systems by forming virtual antenna arrays to exploit spatial diversity gains and improve network performance [1]. The benefits of such networks can be further reaped through optimal power allocation between the source and/or relay nodes, so as to improve different performance criteria. However, doing do requires the knowledge of the channel state information at the relays and/or destination nodes. In time-varying wireless channels, optimal power allocation is a challenging task due to the need for accurate and complete global instantaneous channel state information (CSI). In turn, the power allocation task becomes 
a dynamic problem; requiring adaptive computationally-expensive algorithmic solutions, and excessive communication overheads. In practice, instantaneous CSI is obtained via channel estimation, which introduces estimation errors [2]. Consequently, such dynamic/adaptive algorithms experience significant performance loss, as the CSI may be erroneous and rather outdated, which has detrimental effects on the end-to-end signal-to-noise ratio (SNR), ultimately leading to sub-optimal performance and/or violation of quality-of-service (QoS) constraints [3,4]. Instead, each user should aim at maximizing its average achievable rate, while satisfying a probabilistic QoS specification (i.e. its average rate being not less than its target value [5]). To achieve this, it is imperative to consider alternative means to optimal power allocation with partial CSI, so as to achieve optimal/near-optimal performance while reducing computational complexity and communication overheads [6].

There has been a plethora of research works on power allocation in amplify-and-forward (AF) relay networks; however, most of them assume fixed channel gains or are based on dynamic algorithms with perfect knowledge of instantaneous CSI of all network users and/or relays [7]. For instance, in [8], the authors derive a closed-form optimal power allocation solution for multiple AF relays, with total and individual power constraints. Specifically, the derived solution requires instantaneous CSI. In [9], distributed and centralized power allocation for multiuser multi-relay AF networks is studied. Specifically, the authors consider the problems of maximization of the minimum rate, and the weighted sum-of-rates, which are based on perfect instantaneous CSI knowledge. In [10], a distributed iterative auction-based power allocation mechanism is proposed for deterministic multi-source multi-relay power allocation in relay networks, such that the network sum-rate is maximized. In particular, each source node must obtain complete CSI in order to be able to demand relay power based on the announced relay prices. In [11], the authors study the problem of minimizing the total transmission power subject to an outage constraint, and minimizing the outage probability subject to total transmit powers constraints in multi-hop multibranch AF networks. To be specific, the authors derive asymptotically tight approximations of the received SNR, which are then used to formulate optimization problems using geometric programming (GP). Such GP problems are then transformed into nonlinear convex problems, which can be solved efficiently. In [12], the authors proposed efficient power allocation schemes for multi-source multi-relay AF networks, so as to maximize the network throughput and the minimum end-to-end SNR among the users, and also minimize the total transmit power of all users. Particularly, the proposed schemes are based on GP, which are transformed into equivalent convex optimization problems that can be solved efficiently. Total power minimization subject target symbol error rate (SER) QoS requirement is investigated in [13] for single-source multi-relay AF networks. Particularly, the authors derived closed-form power allocation 
solutions, and proposed power allocation algorithms to prolong network lifetime. Optimal power allocation for instantaneous SNR maximization in multi-hop AF networks under short-term (ST) and long-term (LT) power constraints is studied in [14]. Particularly, the authors illustrate that at sufficiently high SNR values, significant performance gains in terms of outage probability can be achieved with optimal power allocation over uniform power allocation under ST power constraint; while under the LT power constraint, substantial performance gain can be achieved in the low as well as high SNR regime. In [15], power allocation strategies for maximizing the end-to-end SNR, and minimizing the total power consumption while maintaining end-to-end SNR are studied for fixed-gain AF networks in Nakagami- $m$ fading channels. Specifically, the formulated strategies consider the all-participate as well as selective relaying under full and limited feedback, and are solved via convex optimization. Generally speaking, the proposed optimal power allocation solutions/algorithms are based on instantaneous CSI, and this may be not be suitable in time-varying channels [16]. Additionally, the computational complexity and communication overheads involved in dynamic power allocation may not be practical. Therefore, it is essential to consider power allocation strategies that do not relay on instantaneous CSI, and still be suitable for time-varying channels, while satisfying QoS constraints.

In this paper, power allocation over time-varying multi-user multi-relay amplify-and-forward networks is investigated. Particularly, the power allocation problems of network sum-rate maximization, max-min rate and total power minimization are formulated as stochastic optimization problems, subject to QoS constraints (in terms of target rate or outage probability). The stochastic optimization problems are then transformed into optimal asymptotically convex deterministic problems at high enough SNR, where the time-varying rate function of each source-destination pair is replaced by its "time-average" ergodic rate function. Such transformations are based on channel variances only (i.e. partial CSI). Consequently, they can be performed efficiently in an offline manner, as opposed to online power allocation, which requires complete instantaneous CSI and involves computationally-expensive optimization techniques. Simulations results demonstrate that the convex approximations at high enough SNR closely agree with their derived optimal deterministic and dynamic counterparts. It should be noted that in [17], the authors study optimal power allocation for minimizing outage probability in the high SNR regime subject to total power constraint, for the amplify-and-forward, decode-and-forward and distributed space-time coding protocols. Particularly, convex approximations based on mean channel gain information are provided to achieve improvements in the outage probability while achieving significant coding gains. In [18], the authors derive an expression for the ergodic capacity and provide an upper-bound for a single-user multirelay AF network. After that, they propose a novel quasi-optimal power allocation scheme that maximizes 
the upper-bound of the derived ergodic capacity and conclude that the cooperative mode should only be used when the source-to-destination channel gain is worst that that of the relay-to-destination. Hence, to the best of our knowledge, no prior work has considered the formulation and transformation of stochastic sum-rate maximizing and max-min rate power allocation, and total power minimization problems for multi-user multi-relay AF networks into their deterministic representations, and provide asymptotically convex approximations that are solvable with minimal computational complexity.

The remainder of this paper is organized as follows. Section II presents the stochastic and deterministic power allocation problems, while Section III provides the asymptotically convex reformulations. Section IV presents the stochastic total power minimization formulation as well as its deterministic convex approximation. Simulation results are presented in Section V, while conclusions are drawn in Section VI.

\section{Power Allocation Formulations}

\section{A. Network Model}

Consider an orthogonal "time-slotted" cooperative relay network of $N$ source-destination pairs and $K$ AF relay nodes. Each source node $S_{i}$ has transmit power of $P_{S_{i}}(t)$, for $i \in\{1,2, \ldots, N\}$, while each relay $R_{k}$-for $k \in\{1,2, \ldots, K\}$-allocates transmit power $P_{R_{k}, S_{i}}(t)$ to that source node. Let $h_{s_{i}, r_{k}}(t), h_{r_{k}, d_{i}}(t)$ and $h_{s_{i}, d_{i}}(t)$ be the time-varying channel coefficients of the source-relay, relay-destination and sourcedestination links of nodes $S_{i}, R_{k}$ and $D_{i}$, which are modeled as zero-mean complex Gaussian random variables with variances $\sigma_{S_{i}, R_{k}}^{2}, \sigma_{R_{k}, D_{i}}^{2}$ and $\sigma_{S_{i}, D_{i}}^{2}$, respectively. Additionally, each source-destination pair $S_{i}-D_{i}$ is assigned a signature waveform $c_{i}(t)$, which allows multiuser detection at the intended destination node. Waveforms $c_{i}(t)$ and $c_{j}(t)$ have correlation coefficient $\rho_{i, j}$, where $0 \leq \rho_{i, j} \leq 1$ for $i \neq j$, and $\rho_{i, i}=1$. For simplicity, let $\rho_{i, j}=\rho, \forall j \neq i$ [19]. It is assumed that there is a total power constraint $P_{\max }$ per time-slot $t$. Thus, $P_{S_{i}}(t) \leq P_{\max }, \forall i \in\{1,2, \ldots, N\}$, and $\sum_{i=1}^{N} P_{R_{k}, S_{i}}(t) \leq P_{\max }$, $\forall k \in\{1,2, \ldots, K\}$.

Communication between each source-destination pair is performed over $N+K$ time-slots. Particularly, each source node $S_{i}$ is assigned a time-slot to broadcast its data symbol, which is received by each relay and destination node. After that, each relay $R_{k}$-in its assigned time-slot—forms a linearly-coded signal of all received signals and transmits it to the destination nodes, where each destination node performs multiuser detection to separate the different users' data symbols [10,20]. The instantaneous SNR resulting at destination node $D_{i}$ is given by [10] 


$$
\begin{aligned}
\gamma_{i}(t) & =\gamma_{i, i}(t)+\sum_{k=1}^{K} \gamma_{k, i}(t) \\
& =\frac{P_{S_{i}}(t)\left|h_{S_{i}, D_{i}}(t)\right|^{2}}{N_{0}}+\sum_{k=1}^{K} \frac{1}{\varrho N_{0}} \frac{P_{S_{i}}(t) P_{R_{k}, S_{i}}(t)\left|h_{S_{i}, R_{k}}(t)\right|^{2}\left|h_{R_{k}, D_{i}}(t)\right|^{2}}{P_{S_{i}}(t)\left|h_{S_{i}, R_{k}}(t)\right|^{2}+P_{R_{k}, S_{i}}(t)\left|h_{R_{k}, D_{i}}(t)\right|^{2}+N_{0}},
\end{aligned}
$$

Particularly, $N_{0}$ is the noise variance, while $\varrho$ is given by

$$
\varrho=\frac{1+(N-2) \rho}{1+(N-2) \rho-(N-1) \rho^{2}} .
$$

Additionally, one must note that $\gamma_{i, i}(t)$ is an exponential random variable with average rate $\lambda_{S_{i}, D_{i}}=$ $\frac{N_{0}}{P_{S_{i}} \sigma_{S_{i}, D_{i}}^{2}}$.

Remark 1: Due to the strict increasing monotonicity of the SNR terms $\gamma_{i}(t)$ and $\gamma_{k, i}(t)$ in $P_{S_{i}}(t)$, $\forall i \in\{1,2, \ldots, N\}$ and $\forall k \in\{1,2, \ldots, K\}$, then $P_{S_{i}}(t) \triangleq P_{S}=P_{\max }, \forall i \in\{1,2, \ldots, N\}$, and $\forall t \geq 1$.

The achievable rate for each source-destination pair $S_{i}-D_{i}$ is given by [19]

$$
R_{i}\left(\mathbf{P}_{R, S_{i}}(t)\right)=\frac{1}{N+K} \log _{2}\left(1+\gamma_{i, i}(t)+\sum_{k=1}^{K} \gamma_{k, i}(t)\right)
$$

where $\mathbf{P}_{R, S_{i}}(t)=\left[P_{R_{1}, S_{i}}(t), P_{R_{2}, S_{i}}(t), \ldots, P_{R_{K}, S_{i}}(t)\right]^{T}$. Based on Remark 1, the rate function in (3) is a function of $P_{R_{k}, S_{i}}(t), \forall k \in\{1,2, \ldots, K\}$ only.

\section{B. Network Sum-Rate Maximization}

The stochastic network sum-rate power allocation (S-NSR-PA) is formulated as

\section{S-NSR-PA:}

$$
\begin{array}{ll}
\max & \sum_{i=1}^{N} \mathbb{E}\left[R_{i}\left(\mathbf{P}_{R, S_{i}}(t)\right)\right] \\
\text { s.t. } & \sum_{i=1}^{N} P_{R_{k}, S_{i}}(t) \leq P_{\max }, \quad \forall k \in\{1,2, \ldots, K\}, \\
& \mathbb{P}\left[R_{i}\left(\mathbf{P}_{R, S_{i}}(t)\right) \leq R_{T}\right] \leq p_{T}, \quad \forall i \in\{1,2, \ldots, N\}, \\
& P_{R_{k}, S_{i}}(t) \geq 0, \quad \forall k \in\{1,2, \ldots, K\} \text { and } \forall i \in\{1,2, \ldots, N\} .
\end{array}
$$


1) Average Network Sum-Rate: To simplify the analysis, the rate function in (3) can alternatively be expressed as [21]

$$
R_{i}\left(\mathbf{P}_{R, S_{i}}(t)\right)=\frac{1}{(N+K) \ln 2} \int_{0}^{\infty} \frac{e^{-z}}{z}\left(1-e^{-z\left(\gamma_{i, i}(t)+\sum_{k=1}^{K} \gamma_{k, i}(t)\right)}\right) d z,
$$

where the expectation of $R_{i}\left(\mathbf{P}_{R, S_{i}}(t)\right)$ is written as [21]

$$
\mathbb{E}\left[R_{i}\left(\mathbf{P}_{R, S_{i}}(t)\right)\right]=\frac{1}{(N+K) \ln 2} \int_{0}^{\infty} \frac{e^{-z}}{z}\left(1-\mathcal{M}_{i}(z)\right) d z .
$$

Additionally, $\mathcal{M}_{i}(z)$ is given by

$$
\mathcal{M}_{i}(z)=\mathcal{M}_{i, i}(z) \cdot \prod_{k=1}^{K} \mathcal{M}_{k, i}(z)
$$

where $\mathcal{M}_{i, i}(z)$ is given by [1]

$$
\mathcal{M}_{i, i}(z)=\frac{1}{1+z P_{S} \sigma_{S_{i}, D_{i}}^{2} / N_{0}}
$$

while $\mathcal{M}_{k, i}(z)$ is given by [22]

$$
\begin{aligned}
& \mathcal{M}_{k, i}(z)=\frac{16 \lambda_{S_{i}, R_{k}} \lambda_{R_{k}, D_{i}}}{3\left(\lambda_{S_{i}, R_{k}}+\lambda_{R_{k}, D_{i}}+2 \sqrt{\lambda_{S_{i}, R_{k}} \lambda_{R_{k}, D_{i}}}+z\right)^{2}} \times
\end{aligned}
$$

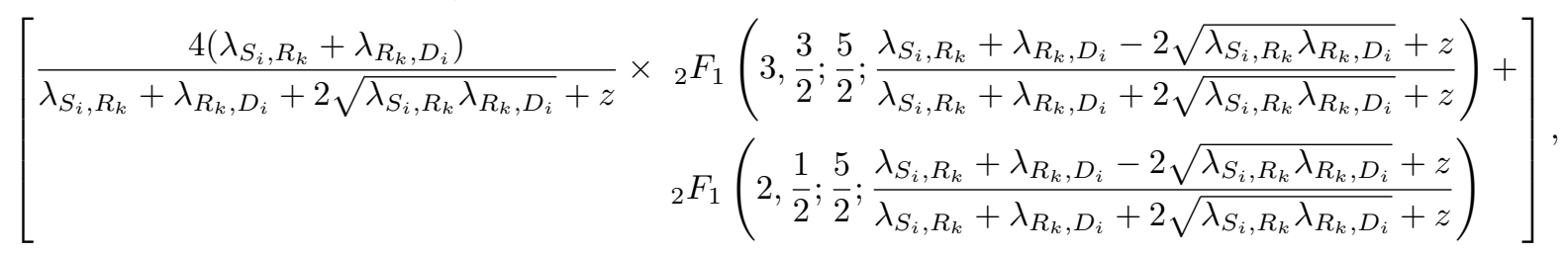

where ${ }_{2} F_{1}(\cdot, \cdot ; \cdot ; \cdot)$ is the hypergeometric function [23], $\lambda_{S_{i}, R_{k}}=\frac{N_{0} \varrho}{P_{S} \sigma_{S_{i}, R_{k}}^{2}}$, and $\lambda_{R_{k}, D_{i}}=\frac{N_{0} \varrho}{P_{R_{k}, S_{i}} \sigma_{R_{k}, D_{i}}^{2}}$. Hence, the approximate average network sum-rate can be shown to be [24, Lemma 1]

$$
\sum_{i=1}^{N} \mathbb{E}\left[R_{i}\left(\mathbf{P}_{R, S_{i}}\right)\right]=\frac{1}{(N+K) \ln 2} \cdot \sum_{m=1}^{M} \frac{\alpha_{m}}{\beta_{m}} \cdot\left[\sum_{i=1}^{N}\left[1-\mathcal{M}_{i}\left(\beta_{m}, \mathbf{P}_{R, S_{i}}\right)\right]\right],
$$

where $\beta_{m}$ and $\alpha_{m}$ are the abscissas and weight factors of the Laguerre polynomial, respectively (as given in [25, Table 25.9]).

2) Outage Probability: The approximate outage probability is expressed as [5]

$$
P_{\mathrm{Out}, i}\left(\mathbf{P}_{R, S_{i}}\right) \simeq \sum_{k=1}^{K+1}\left(\prod_{m=1, m \neq k}^{K+1} \frac{\lambda_{S_{i}, R_{m}, D_{i}}}{\lambda_{S_{i}, R_{m}, D_{i}}-\lambda_{S_{i}, R_{k}, D_{i}}}\right)\left(1-e^{-\lambda_{S_{i}, R_{k}, D_{i}} \bar{R}_{T}}\right),
$$

where $\bar{R}_{T}=2^{(N+K) R_{T}}-1$, and 


$$
\lambda_{S_{i}, R_{k}, D_{i}}=\left\{\begin{array}{ll}
\lambda_{S_{i}, R_{k}}+\lambda_{R_{k}, D_{i}}=\varrho N_{0} \cdot \frac{P_{S} \sigma_{S_{i}, R_{k}}^{2}+P_{R_{k}, S_{i}} \sigma_{R_{k}, S_{i}}^{2}}{P_{S} P_{R_{k}, S_{i}} \sigma_{S_{i}, R_{k}}^{2} \sigma_{R_{k}, D_{i}}^{2},} & \text { if } k \neq K+1 \\
\lambda_{S_{i}, D_{i}}=\frac{N_{0}}{P_{S} \sigma_{S_{i}, D_{i}}^{2}}, & \text { if } k=K+1
\end{array} .\right.
$$

Therefore, the deterministic power allocation optimization problem is expressed as

D-NSR-PA:

$$
\begin{array}{ll}
\max & \frac{1}{(N+K) \ln 2} \cdot \sum_{m=1}^{M} \frac{\alpha_{m}}{\beta_{m}} \cdot\left[\sum_{i=1}^{N}\left[1-\mathcal{M}_{(i)}\left(\beta_{m}, \mathbf{P}_{R, S_{i}}\right)\right]\right] \\
\text { s.t. } & \sum_{i=1}^{N} P_{R_{k}, S_{i}} \leq P_{\max }, \quad \forall k \in\{1,2, \ldots, K\}, \\
& P_{\text {Out }, i}\left(\mathbf{P}_{R, S_{i}}\right) \leq p_{T}, \quad \forall i \in\{1,2, \ldots, N\}, \\
& P_{R_{k}, S_{i}} \geq 0, \quad \forall k \in\{1,2, \ldots, K\} \text { and } \forall i \in\{1,2, \ldots, N\} .
\end{array}
$$

\section{Max-Min Rate}

The stochastic max-min rate power allocation (S-MMR-PA) problem is expressed as

\section{S-MMR-PA:}

$$
\begin{array}{ll}
\max & \min _{i \in\{1,2, \ldots, N\}} \mathbb{E}\left[R_{i}\left(\mathbf{P}_{R, S_{i}}(t)\right)\right] \\
\text { s.t. } & \sum_{i=1}^{N} P_{R_{k}, S_{i}}(t) \leq P_{\max }, \quad \forall k \in\{1,2, \ldots, K\}, \\
& \mathbb{P}\left[R_{i}\left(\mathbf{P}_{R, S_{i}}(t)\right) \leq R_{T}\right] \leq p_{T}, \quad \forall i \in\{1,2, \ldots, N\}, \\
& P_{R_{k}, S_{i}}(t) \geq 0, \quad \forall k \in\{1,2, \ldots, K\} \text { and } \forall i \in\{1,2, \ldots, N\} .
\end{array}
$$

The above problem can be transformed into a deterministic optimization problem as

\section{D-MMR-PA:}

$$
\begin{array}{ll}
\max & \eta \\
\text { s.t. } & \sum_{i=1}^{N} P_{R_{k}, S_{i}} \leq P_{\max }, \quad \forall k \in\{1,2, \ldots, K\}, \\
& \frac{1}{(N+K) \ln 2} \cdot \sum_{m=1}^{M} \frac{\alpha_{m}}{\beta_{m}} \cdot\left[1-\mathcal{M}_{(i)}\left(\beta_{m}, \mathbf{P}_{R, S_{i}}\right)\right] \geq \eta, \quad \forall i \in\{1,2, \ldots, N\}, \\
& P_{\mathrm{Out}, i}\left(\mathbf{P}_{R, S_{i}}\right) \leq p_{T}, \quad \forall i \in\{1,2, \ldots, N\}, \\
& P_{R_{k}, S_{i}} \geq 0, \quad \forall k \in\{1,2, \ldots, K\} \text { and } \forall i \in\{1,2, \ldots, N\}, \\
& \eta \geq 0 .
\end{array}
$$


Remark 2: Problems D-NSR-PA and D-MMR-PA are non-convex, due to the non-convexity of the rate function and outage probability of each source-destination pair. Hence, they can only be solved efficiently using a global optimization software package.

\section{Asymptotically Convex Power Allocation}

This section provides approximate solutions to the network sum-rate maximization and max-min rate deterministic power allocation problems at high enough SNR.

The MGF function $\mathcal{M}_{(i)}(z)$ in (7) —at high enough SNR—can be approximated as [1]

$$
\overline{\mathcal{M}}_{(i)}(z)=\overline{\mathcal{M}}_{i, i}(z) \cdot \prod_{k=1}^{K} \overline{\mathcal{M}}_{k, i}(z)
$$

where

$$
\overline{\mathcal{M}}_{i, i}(z) \simeq \frac{N_{0}}{z P_{S} \sigma_{S_{i}, D_{i}}^{2}}
$$

while

$$
\overline{\mathcal{M}}_{k, i}(z) \simeq \frac{\varrho N_{0}}{z} \cdot \frac{P_{S} \sigma_{S_{i}, R_{k}}^{2}+P_{R_{k}, S_{i}} \sigma_{R_{k}, S_{i}}^{2}}{P_{S} P_{R_{k}, S_{i}} \sigma_{S_{i}, R_{k}}^{2} \sigma_{R_{k}, D_{i}}^{2}} .
$$

Note that $\overline{\mathcal{M}}_{i, i}(z)$ in (17) is independent of $P_{R_{k}, S_{i}}$.

The outage probability is tightly approximated and upper-bounded at high enough SNR as [5]

$$
\bar{P}_{\text {Out }, i}\left(\mathbf{P}_{R, S_{i}}\right) \simeq \frac{\left(\bar{R}_{T}\right)^{K+1}}{(K+1) !} \cdot \frac{N_{0}}{P_{S} \sigma_{S_{i}, D_{i}}^{2}} \cdot \prod_{k=1}^{K} \varrho N_{0} \frac{P_{S} \sigma_{S_{i}, R_{k}}^{2}+P_{R_{k}, S_{i}} \sigma_{R_{k}, S_{i}}^{2}}{P_{S} P_{R_{k}, S_{i}} \sigma_{S_{i}, R_{k}}^{2} \sigma_{R_{k}, D_{i}}^{2}} .
$$

Remark 3: It can be verified that $\overline{\mathcal{M}}_{(i)}(z)$ and $\bar{P}_{\text {Out }, i}$ are convex in $P_{R_{k}, S_{i}}, \forall i \in\{1,2, \ldots, N\}$ and $\forall k \in\{1,2, \ldots, K\}[5]$.

At high enough SNR, the deterministic network sum-rate maximizing power allocation problem becomes

\section{A-D-NSR-PA:}

$$
\begin{array}{ll}
\max & \frac{1}{(N+K) \ln 2} \cdot \sum_{m=1}^{M} \frac{\alpha_{m}}{\beta_{m}} \cdot\left[\sum_{i=1}^{N}\left[1-\overline{\mathcal{M}}_{(i)}\left(\beta_{m}, \mathbf{P}_{R, S_{i}}\right)\right]\right] \\
\text { s.t. } & \sum_{i=1}^{N} P_{R_{k}, S_{i}} \leq P_{\max }, \quad \forall k \in\{1,2, \ldots, K\}, \\
& \bar{P}_{\text {Out }, i}\left(\mathbf{P}_{R, S_{i}}\right) \leq p_{T}, \quad \forall i \in\{1,2, \ldots, N\}, \\
& P_{R_{k}, S_{i}} \geq 0, \quad \forall k \in\{1,2, \ldots, K\} \text { and } \forall i \in\{1,2, \ldots, N\} .
\end{array}
$$




\section{A. Max-Min Rate}

Similarly, the approximate deterministic max-min rate power allocation problem at high enough SNR is expressed as

\section{A-D-MMR-PA:}

$$
\begin{array}{ll}
\max & \eta \\
\text { s.t. } & \sum_{i=1}^{N} P_{R_{k}, S_{i}} \leq P_{\max }, \quad \forall k \in\{1,2, \ldots, K\}, \\
& \frac{1}{(N+K) \ln 2} \cdot \sum_{m=1}^{M} \frac{\alpha_{m}}{\beta_{m}} \cdot\left[1-\overline{\mathcal{M}}_{(i)}\left(\beta_{m}, \mathbf{P}_{R, S_{i}}\right)\right] \geq \eta, \quad \forall i \in\{1,2, \ldots, N\}, \\
& \bar{P}_{\text {Out }, i}\left(\mathbf{P}_{R, S_{i}}\right) \leq p_{T}, \quad \forall i \in\{1,2, \ldots, N\}, \\
& P_{R_{k}, S_{i}} \geq 0, \quad \forall k \in\{1,2, \ldots, K\} \text { and } \forall i \in\{1,2, \ldots, N\}, \\
& \eta \geq 0 .
\end{array}
$$

Remark 4: Problems A-D-NSR-PA and A-D-MMR-PA are convex optimization problems and thus can be efficiently solved using any standard convex optimization software package.

\section{Total Power Minimization}

The stochastic total power minimization (S-TPM) problem is formulated as

\section{S-TPM:}

$$
\begin{array}{ll}
\min & \sum_{k=1}^{K} \sum_{i=1}^{N} P_{R_{k}, S_{i}}(t) \\
\text { s.t. } & \sum_{i=1}^{N} P_{R_{k}, S_{i}}(t) \leq P_{\max }, \quad \forall k \in\{1,2, \ldots, K\}, \\
& \mathbb{E}\left[R_{i}\left(\mathbf{P}_{R, S_{i}}(t)\right)\right] \geq R_{T}, \quad \forall i \in\{1,2, \ldots, N\}, \\
& P_{R_{k}, S_{i}}(t) \geq 0, \quad \forall k \in\{1,2, \ldots, K\} \text { and } \forall i \in\{1,2, \ldots, N\},
\end{array}
$$

which is transformed into a deterministic problem as

\section{D-TPM:}

$$
\begin{aligned}
\min & \sum_{k=1}^{K} \sum_{i=1}^{N} P_{R_{k}, S_{i}} \\
\text { s.t. } & \sum_{i=1}^{N} P_{R_{k}, S_{i}} \leq P_{\max }, \quad \forall k \in\{1,2, \ldots, K\},
\end{aligned}
$$




$$
\begin{aligned}
& \frac{1}{(N+K) \ln 2} \cdot \sum_{m=1}^{M} \frac{\alpha_{m}}{\beta_{m}} \cdot\left[1-\mathcal{M}_{(i)}\left(\beta_{m}, \mathbf{P}_{R, S_{i}}\right)\right] \geq R_{T}, \quad \forall i \in\{1,2, \ldots, N\}, \\
& P_{R_{k}, S_{i}} \geq 0, \quad \forall k \in\{1,2, \ldots, K\} \text { and } \forall i \in\{1,2, \ldots, N\},
\end{aligned}
$$

which is non-convex due to the non-convexity of the rate function in (23b). However, by using (16), the approximate deterministic problem at high enough SNR is written as

\section{A-D-TPM:}

$$
\begin{array}{ll}
\min & \sum_{k=1}^{K} \sum_{i=1}^{N} P_{R_{k}, S_{i}} \\
\text { s.t. } & \sum_{i=1}^{N} P_{R_{k}, S_{i}} \leq P_{\max }, \quad \forall k \in\{1,2, \ldots, K\}, \\
& \frac{1}{(N+K) \ln 2} \cdot \sum_{m=1}^{M} \frac{\alpha_{m}}{\beta_{m}} \cdot\left[1-\overline{\mathcal{M}}_{(i)}\left(\beta_{m}, \mathbf{P}_{R, S_{i}}\right)\right] \geq R_{T}, \quad \forall i \in\{1,2, \ldots, N\}, \\
& P_{R_{k}, S_{i}} \geq 0, \quad \forall k \in\{1,2, \ldots, K\} \text { and } \forall i \in\{1,2, \ldots, N\},
\end{array}
$$

which is now a convex optimization problem.

\section{Simulation Results}

This section evaluates the formulated optimal deterministic power allocation problems and compares them with the approximated problems and equal power allocation (EPA). Moreover, dynamic optimal power allocation (Dyn-OPA) is also compared and formulated as

\section{Dyn-OPA:}

$$
\begin{array}{lll}
\max & f\left(R_{1}\left(\mathbf{P}_{R, S_{1}}(t)\right), R_{2}\left(\mathbf{P}_{R, S_{2}}(t)\right), \ldots, R_{N}\left(\mathbf{P}_{R, S_{N}}(t)\right)\right) \\
\text { s.t. } & \sum_{i=1}^{N} P_{R_{k}, S_{i}}(t) \leq P_{\max }, & \forall k \in\{1,2, \ldots, K\}, \\
& R_{i}\left(\mathbf{P}_{R, S_{i}}(t)\right) \geq R_{T}, & \forall i \in\{1,2, \ldots, N\}, \\
& P_{R_{k}, S_{i}}(t) \geq 0, \quad \forall k \in\{1,2, \ldots, K\} \text { and } \forall i \in\{1,2, \ldots, N\} .
\end{array}
$$

where

$$
f\left(R_{1}\left(\mathbf{P}_{R, S_{1}}(t)\right), R_{2}\left(\mathbf{P}_{R, S_{2}}(t)\right), \ldots, R_{N}\left(\mathbf{P}_{R, S_{N}}(t)\right)\right)= \begin{cases}\sum_{i=1}^{N} R_{i}\left(\mathbf{P}_{R, S_{i}}(t)\right), & \text { for NSR } \\ \min _{i \in\{1,2, \ldots, N\}} R_{i}\left(\mathbf{P}_{R, S_{i}}(t)\right), & \text { for MMR. }\end{cases}
$$


Additionally, the dynamic optimal total power minimization (Dyn-O-TPM) problem is expressed as ${ }^{1}$

\section{Dyn-O-TPM:}

$$
\begin{array}{lll}
\min & \sum_{k=1}^{K} \sum_{i=1}^{N} P_{R_{k}, S_{i}}(t) & \\
\text { s.t. } & \sum_{i=1}^{N} P_{R_{k}, S_{i}}(t) \leq P_{\max }, & \forall k \in\{1,2, \ldots, K\}, \\
& R_{i}\left(\mathbf{P}_{R, S_{i}}(t)\right) \geq R_{T}, & \forall i \in\{1,2, \ldots, N\}, \\
& P_{R_{k}, S_{i}}(t) \geq 0, \quad \forall k \in\{1,2, \ldots, K\} \text { and } \forall i \in\{1,2, \ldots, N\} .
\end{array}
$$

The network topology is illustrated in Fig. 1, and the channel gain between any two nodes is given by $\sigma^{2}=d^{-\nu}$, where $d$ and $\nu$ are the inter-node distance and path-loss exponent, respectively. Moreover, the simulations assume $P_{\max }=150 \mathrm{~mW}, \nu=2.5, \rho=0.15$, and $R_{T}=0.5 \mathrm{bits} / \mathrm{s} / \mathrm{Hz}$, and are averaged over $10^{6}$ independent runs with randomly generated channel coefficients that change every time-slot ${ }^{2}$.

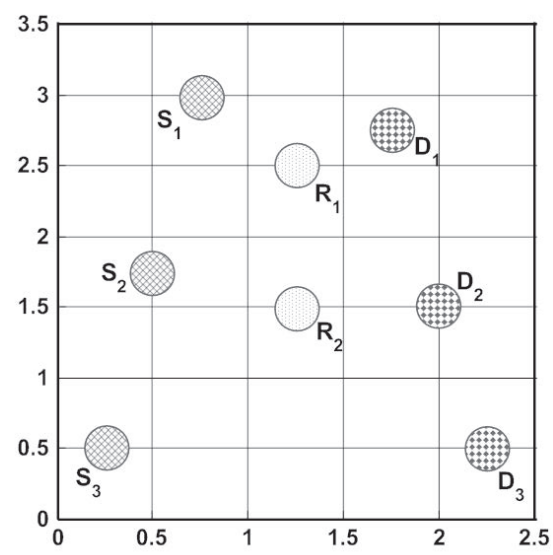

Fig. 1. Network Topology

Fig. 2 demonstrates the average rate per source-destination pair as well as the average network sum-rate under relay equal power allocation (EPA) and for different orders of approximation $M$. One can see that source-destination pair $S_{1}-D_{1}$ achieves the highest rate, when compared with pairs $S_{2}-D_{2}$ and $S_{3}-D_{3}$. This is explained by noting that nodes $S_{1}$ and $D_{1}$ are relatively closer to each other than the nodes of the other two pairs. Additionally, nodes $S_{1}$ and $D_{1}$ are relatively closer to relays $R_{1}$ and $R_{2}$ than the other

\footnotetext{
${ }^{1}$ The deterministic optimal, approximate and dynamic optimization problems are solved via MIDACO, with tolerance set to $0.0001[26]$.

${ }^{2}$ Dynamic optimal power allocation and total power minimization are performed at the end of the broadcasting phase (i.e. before the cooperation phase), and assumed to be achieved via a centralized controller with perfect CSI.
} 


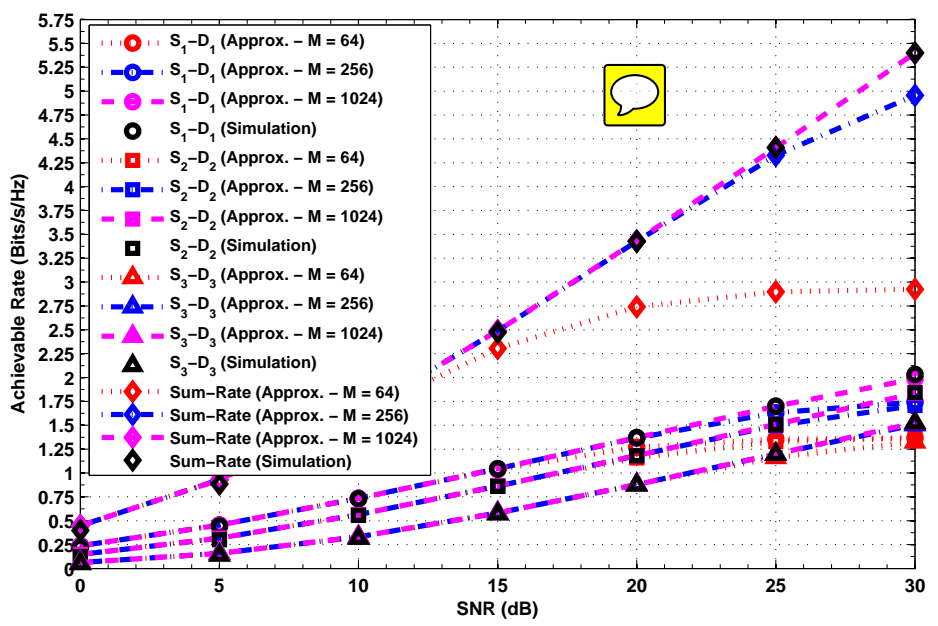

Fig. 2. Achievable Rate: Approximation vs. Simulation - EPA

source/destination nodes, which implies less path-loss and channel noise. This also explains why the pair $S_{3}-D_{3}$ achieves the lowest average rate. It is also clear that increasing the order of approximation $M$ improves the accuracy of the average rate of each source-destination pair, in comparison with the simulated rates. For example, $M=256$ suffices for accurate average rate approximation at $\mathrm{SNR}=20$ $\mathrm{dB}$; while $M=1024$ is sufficient at SNR $=30 \mathrm{~dB}$ (i.e. higher values of SNR require greater values of $M)$.

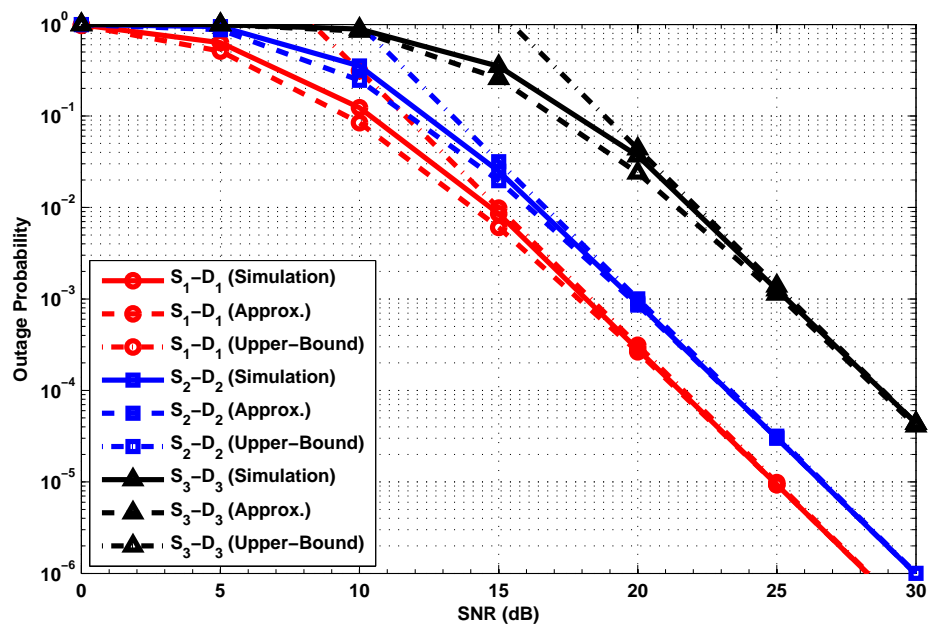

Fig. 3. Outage Probability - EPA

Fig. 3 illustrates the simulated outage probability per source-destination pair under EPA, in comparison with the theoretical approximate and upper-bound outage probabilities, as given by (13) and (21), respectively. Clearly, the theoretical approximate and upper-bound outage probabilities agree with their 
simulated counterpart at $\mathrm{SNR}=30 \mathrm{~dB}$, for all source-destination pairs. Moreover, the pair $S_{3}-D_{3}$ achieves the highest outage probability of $4 \times 10^{-5}$, which agrees with the observation that this pair achieves the lowest average rate.

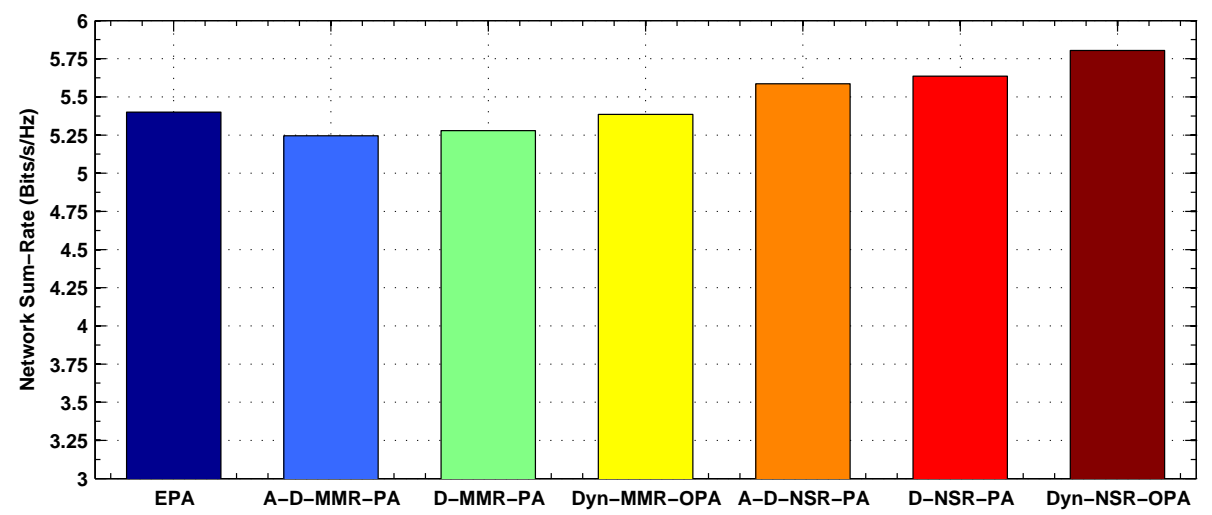

Fig. 4. Comparison of Network Sum-Rate $-p_{T}=10^{-5}, \mathrm{SNR}=30 \mathrm{~dB}$ and $M=1024$

In Fig. 4, the average network sum-rate of the different power allocation problems are compared at $\mathrm{SNR}=30 \mathrm{~dB}$, when $p_{T}=10^{-5}$ and $M=1024$. It is clear that the convex approximate problems A-D-MMR-PA and A-D-NSR-PA agree with their optimal counterparts D-MMR-PA and D-NSR-PA to within 0.03 bits/s/Hz. Moreover, the average network sum-rate under the NSR-PA problems is higher than their MMR-PA counterparts. Additionally, it is evident that the network sum-rates resulting from the Dyn-MMR-OPA and Dyn-NSR-OPA problems are superior their deterministic counterparts, and this is due to the fact that they relay on complete instantaneous CSI, rather than partial CSI. Lastly, one can see that the EPA is superior to the deterministic MMR-PA problems and yields almost the same network sum-rate as the dynamic MMR-PA problem. This is because the MMR-PA problems tend to make the rates of the different source nodes equal, which poses a tradeoff between the network sum-rate and fairness.

Fig 5a presents the average rate per source-destination pair under the different power allocation problems, where it can be seen that the rates are almost equal under the MMR-PA problems, as expected. On the other hand, the pair $S_{1}-D_{1}$ achieves the highest average rate under the NSR-PA problems, as noted before. Moreover, the dynamic power allocation problems yield higher rate per source-destination pair than their deterministic counterparts. Fig. $5 \mathrm{~b}$ illustrates the outage probability per source-destination pair, where it is evident that all the power allocation problems closely satisfy the target outage probability $p_{T} \lesssim 10^{-5}$ for all source-destination pairs, except for the pair $S_{3}-D_{3}$ under the EPA (as noted in Fig. 3). Finally, the NSR-PA problems achieve the lowest outage probability while satisfying the target outage probability $p_{T}$ for all pairs, when compared with the other problems. 
(a) Achievable Rate

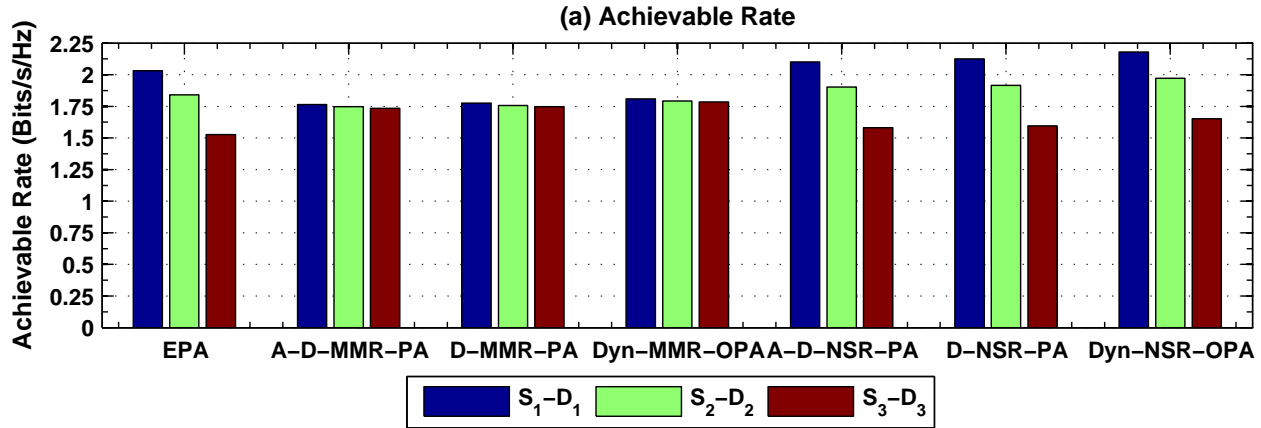

(b) Outage Probability

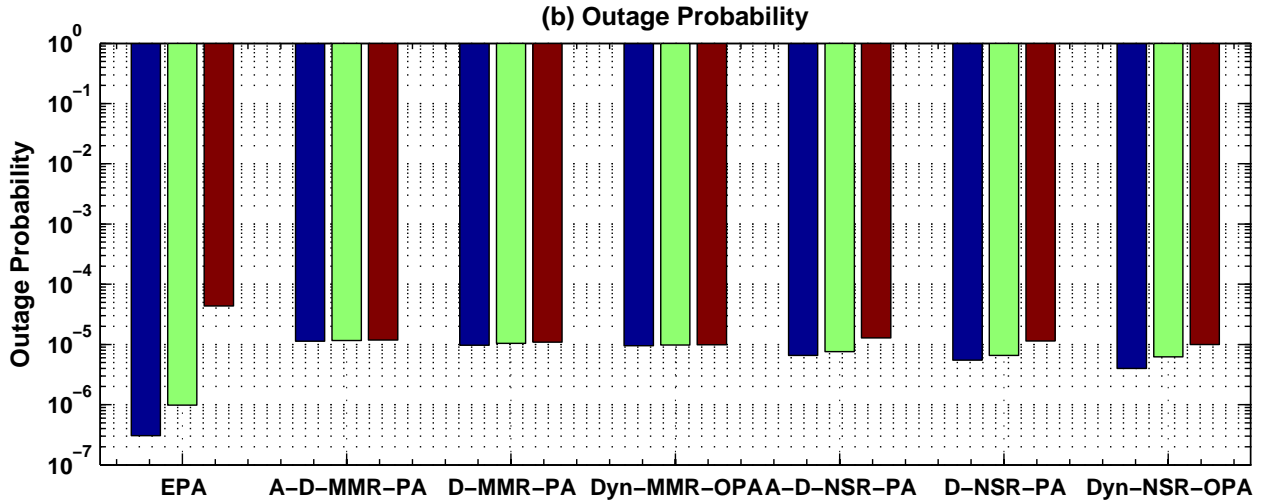

Fig. 5. Comparison of (a) Achievable Rate, and (b) Outage Probability of Each Source-Destination Pair $-p_{T}=10^{-5}$, SNR $=$ $30 \mathrm{~dB}$ and $M=1024$

(a) $R_{T}=1.5 \mathrm{Bits} / \mathrm{s} / \mathrm{Hz}$

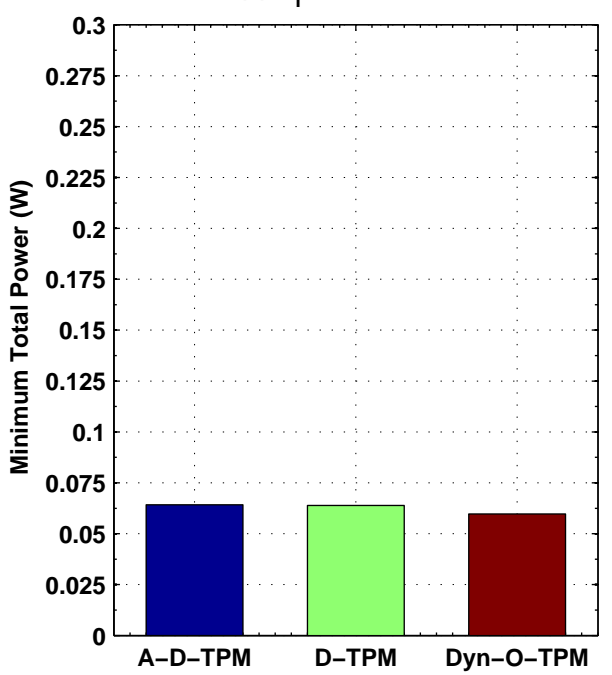

(b) $R_{\mathrm{T}}=1.6 \mathrm{Bits} / \mathrm{s} / \mathrm{Hz}$

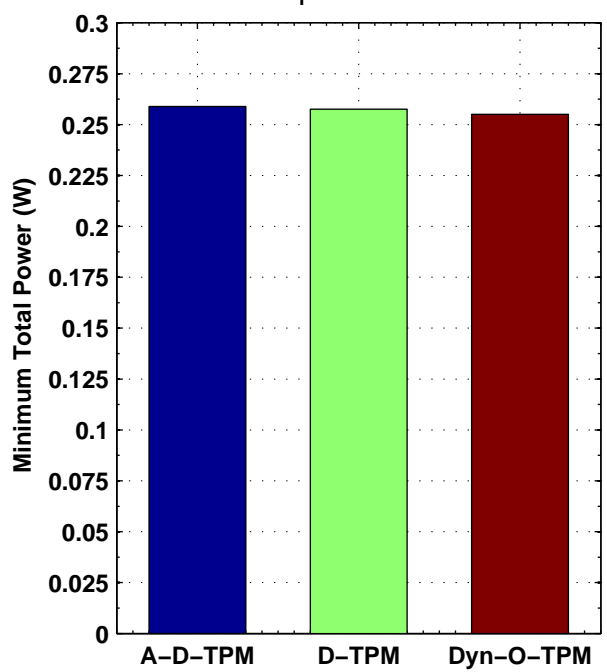

Fig. 6. Minimum Total Power for (a) $R_{T}=1.5 \mathrm{Bits} / \mathrm{s} / \mathrm{Hz}$, and (b) $R_{T}=1.6 \mathrm{Bits} / \mathrm{s} / \mathrm{Hz}-\mathrm{SNR}=30 \mathrm{~dB}$ and $M=1024$

Fig. 6 demonstrates the minimum total power required to satisfy a target rate $R_{T}$ under the exact deterministic total power minimizing problem D-TPM, its asymptotically convex counterpart A-D-TPM, 
and dynamic optimal total power minimizing problem Dyn-O-TPM. Clearly, one can see that for the deterministic problems for target rates $R_{T}=1.5$ and $R_{T}=1.6 \mathrm{bits} / \mathrm{s} / \mathrm{Hz}$, the minimum total power is almost equal, with negligible difference. Additionally, it can be seen that the minimum total power required to satisfy the target rate of $R_{T}=1.5 \mathrm{bits} / \mathrm{s} / \mathrm{Hz}$ (see Fig. 6a) is less than that when $R_{T}=1.6$ bits/s/Hz (see Fig. 6b) under the different problems. This is because the lower the target rate $R_{T}$ is, the smaller the amount of power required to satisfy it. Finally, the Dyn-O-TPM achieves the lowest minimum total power among the different problems and under both target rates. Again, this is attributed to the utilization of complete CSI for total power minimization.

(a) $R_{\mathrm{T}}=1.5 \mathrm{Bits} / \mathrm{s} / \mathrm{Hz}$

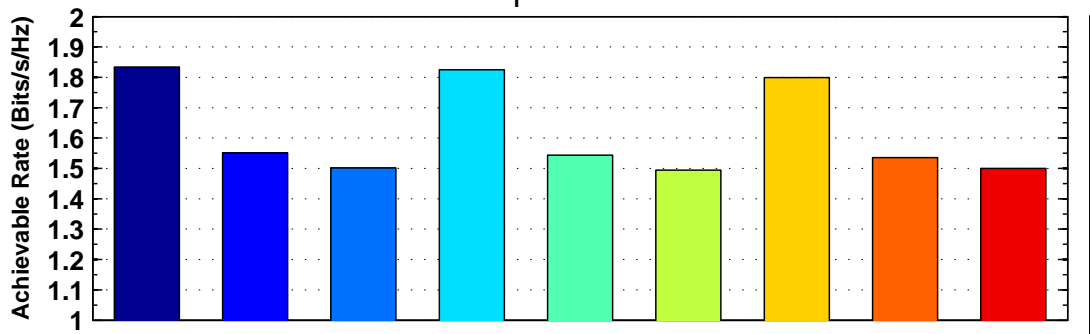

(b) $R_{T}=1.6 \mathrm{Bits} / \mathrm{s} / \mathrm{Hz}$

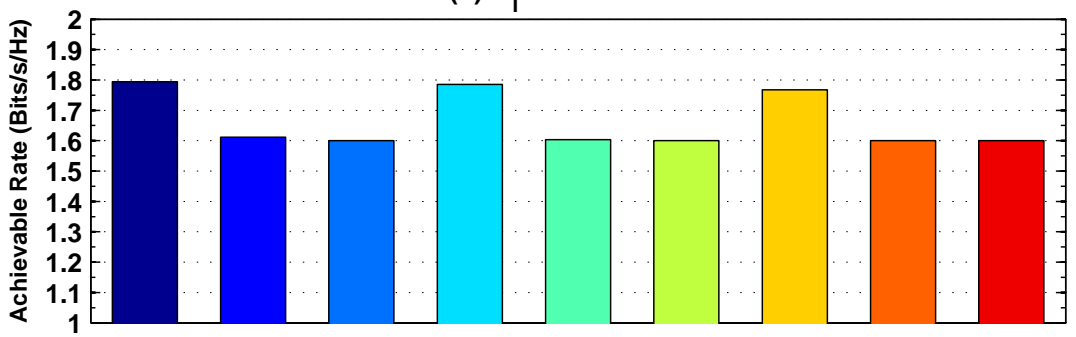

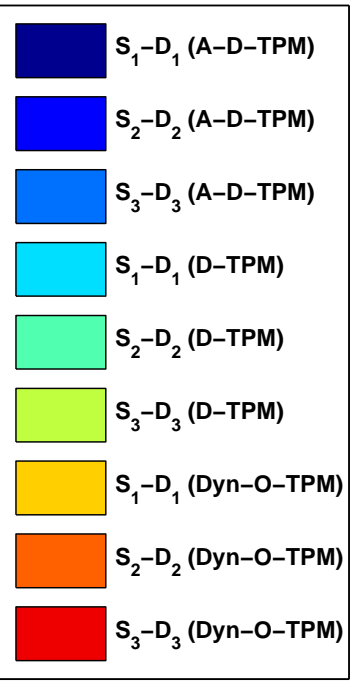

Fig. 7. Achievable Rate Per Source-Destination Pair for (a) $R_{T}=1.5 \mathrm{Bits} / \mathrm{s} / \mathrm{Hz}$, and (b) $R_{T}=1.6 \mathrm{Bits} / \mathrm{s} / \mathrm{Hz}-\mathrm{SNR}=30 \mathrm{~dB}$ and $M=1024$

In Fig. 7, the achievable rates per source-destination pair for different target rates under problems D-TPM, A-D-TPM and Dyn-O-TPM are shown. Specifically, in Fig. 7a (Fig. 7b), one can see that all source-destination pairs satisfy the target rate $R_{T}=1.5 \mathrm{bits} / \mathrm{s} / \mathrm{Hz}\left(R_{T}=1.6 \mathrm{bits} / \mathrm{s} / \mathrm{Hz}\right)$. Additionally, one can see that the achievable rate of the pair $S_{1}-D_{1}$ when $R_{T}=1.5 \mathrm{bit} / \mathrm{s} / \mathrm{Hz}$ is higher than that when $R_{T}=1.6 \mathrm{bits} / \mathrm{s} / \mathrm{Hz}$. This is attributed to the fact that some of the transmit power allocated to the pair $S_{1}-D_{1}$ when $R_{T}=1.5$ bits/s/Hz is re-allocated to pairs $S_{2}-D_{2}$ and $S_{3}-D_{3}$ in order for them to satisfy the higher target rate of $R_{T}=1.6 \mathrm{bits} / \mathrm{s} / \mathrm{Hz}$, which in turn results in a dip to the rate of the $S_{1}-D_{1}$ pair. Also, one can see that the resulting rates of each source-destination pair under the D-TPM problem is only marginally less than those of the A-D-TPM problem, since the total power under the former problem 
is marginally less than the latter problem (see Fig. 6). However, all source-destination pairs satisfy the target rates. This negligible difference is due to the asymptotic approximation. Finally, the rate per source-destination pair under the Dyn-O-TPM problem is less than their deterministic counterparts, since it requires the least amount of power to satisfy the target rates while relaying on complete instantaneous CSI.

The presented results demonstrate that the approximated average rate function of each source-destination pair is highly dependent on the order of approximation $M$. In fact, $M$ must be large enough to yield faithful representation of the average rate of each source-destination pair. Thus, it is arguable that large values of $M$ may entail complex computations and introduce significant delays. However, the derived approximations at high enough SNR yield convex optimization problems, which have polynomial-time complexity, and thus can be solved efficiently. Moreover, it is noteworthy that the optimal deterministic power allocation problems took much longer to converge than the convex approximated ones. One must also keep in mind that these approximate problems are only solved once and in an offline manner, in the network set-up phase. Therefore, they can be solved with minimal computation complexity, without introducing unnecessary communication overheads.

\section{CONCLUSIONS}

In this paper, power allocation for time-varying multi-user multi-relay amplify-and-forward networks is studied. Specifically, stochastic optimization for network sum-rate maximization, max-min rate and total power minimization problems - subject to QoS constraints— have been formulated and transformed into deterministic asymptotically convex problems at high enough SNR. Simulation results illustrate that the convex approximated problems closely agree with their optimal deterministic and dynamic counterparts.

Some potential future research directions are as follows. The work in hand assumes that the transformed deterministic approximate problems are solved via a centralized controller or performed at a source node. However, in practical ad-hoc wireless networks, it may be necessary to solve such problems in a distributed fashion. It may be possible to utilize game-theoretic approaches or primal/dual-decomposition techniques to derive closed-form power allocation solutions, such that each source-destination pair can determine its relay power demands to maximize the network sum-rate or minimum rate, or minimize the total relay transmit power. Moreover, this work implicitly assumes that the channel variances are perfectly known. However, in practical networks, channel gains must be estimated, and thus are error-prone. Therefore, the effect of imperfect estimates of channel gains on the discussed power allocation problems must be quantified. 


\section{REFERENCES}

[1] Liu, K. J. R., Sadek, A. K., Su, W., and Kwasinski, A.: 'Cooperative communications and networking' (Cambridge University Press, 2008).

[2] Ibrahim, A. S., and Liu, K. J. R.: 'Mitigating channel estimation error with timing synchronization tradeoff in cooperative communications', 2010, 58, (1), pp. 337-348.

[3] Yi, Z., and Kim, I.-M.: 'Joint optimization of relay precoders and decoders with partial channel side information in cooperative networks', IEEE Journal on Selected Ares in Communications, 2007, 25, (2), pp. 447-457.

[4] Quek, T. Q. S., Win, M. Z., and Chiani, M.: 'Robust power allocation algorithms for wireless relay networks', IEEE Transactions on Communications, 2010, 58, (7), pp. 1931-1938.

[5] Seddik, K. G., Sadek, A. K., Su, W., and Liu, K. J. R.: 'Outage analysis and optimal power allocation for multimode relay networks', IEEE Signal Processing Letters, 2007, 14, (6), pp. 377-380.

[6] Pham, T. T., Nguyen, H. H., and Tuan, H. D.: 'Power allocation in orthogonal wireless relay networks with partial channel state information', IEEE Transactions on Signal Processing, 2010, 58, (2), pp. 869-878.

[7] Guo, W., Chatzigeorgiou, I., Wassell, I. J., and Carrasco, R.: 'Survey and analysis of power control for collaborative networks'. Proc. of IEEE 2010 7th International Symposium on Communication Systems, Networks, and Digital Signal Processing (CSNDSP), Newcastle, United Kingdom, 2010, pp. 913-918.

[8] Zhao, Y., Adve, R., and Lim, T. J.: 'Improving amplify-and-forward relay networks: optimal power allocation versus selection', IEEE Transactions on Wireless Communications, 2007, 6, (8), pp. 3114-3123.

[9] Phan, K. T., Le, L. B., Vorobyov, S. A., and Ngoc, T. L. N.: 'Power allocation and admission control in multiuser relay networks via convex programming: centralized and distributed schemes', EURASIP Journal on Wireless Communications and Networking, 2009, DOI: 10.1155/2009/901965.

[10] Baidas, M. W., and MacKenzie, A. B.: 'An auction mechanism for power allocation in multi-source multi-relay cooperative wireless networks', IEEE Transactions on Wireless Communications, 2012, 11, (9), pp. 3250-3260.

[11] Mohammadi, M., Ardebilipour, M., Mobini, Z., and Zadeh, R. A. S.: 'Performance analysis and power allocation for multi-hop multi-branch amplify-and-forward cooperative networks over generalized fading channels', EURASIP Journal on Wireless Communications and Networking, 2013, 160, DOI: 10.1186/1687-1499-2013-160.

[12] Phan, K. T., Le-Ngoc, T., Vorobyov, S. A., and Telambura, C.: 'Power allocation in wireless relay networks: a geometric programming-based approach'. Proc. of IEEE Global Communications Conference (GLOBECOM), New Orleans, LO, United States, Dec. 2008, pp. 1-5.

[13] Maham, B., Hjorungnes, A., and Debbah, M.: 'Power allocations in minimum-energy SER constrained cooperative networks', Annales des Telecomunications, 2009, 64, (7), pp. 545-555.

[14] Farhadi, G., and Beaulieu N. C.: 'Power-optimized amplify-and-forward multi-hop relaying systems', IEEE Transactions on Wireless Communications, 2009, 8, (9), pp. 4634-4643.

[15] Zafar, A., Radaydeh, R. M., Chen, Y., and Alouini, M. S.: 'Power allocation strategies for fixed gain half-duplex amplifyand-forward relaying in Nakagami-m fading', IEEE Transactions on Wireless Communications, 2014, 13, (1), pp. 159-173.

[16] Holliday, T., Goldsmith, A., Glynn, P. and Bambos, N.: 'Distributed power and admission control for time-varying wireless networks'. Proc. of IEEE Global Communications Conference (GLOBECOM), Dallas, Texas, United States, Nov 2004, pp. $768-774$.

[17] Annavajjala, R., Cosman, P. C., Milstein, L. B.: 'Statistical channel knowledge-based optimum power allocation for relaying protocols in the high SNR regime', IEEE Journal on Selected Areas in Communications, 2007, 25, (2), pp. $292-305$. 
[18] Lin, F., Luo, T., and Jiang, T.: 'Quasi-optimal power allocation based on ergodic capacity for wireless relay networks', Wireless Communications and Mobile Computing, 2013, 13, (6), pp. 619-632.

[19] Baidas, M. W., and MacKenzie, A. B.: 'Many-to-many space-time network coding for amplify-and-forward cooperative networks: node selection and performance analysis', EURASIP Journal on Wireless Communications and Networking, 2014, 48, DOI: 10.1186/1687-1499-2014-48.

[20] Lai, H. Q., and Liu, K. J. R.: 'Space-time network coding', IEEE Transactions on Signal Processing, 2011, 59, (4), pp. 1706-1718.

[21] Hamdi, K. A.: 'Capacity of MRC on correlated Rician fading chanels', IEEE Transactions on Communications, 2008, 56, (5), pp. 708-711.

[22] Hasna, M. O, and Alouini, M. S.: 'End-to-end performance of transmission systems with relays over Rayleigh fading channels', IEEE Transactions on Wireless Communications, 2003, 2, (6), pp. 1126-1131.

[23] Gradshteyn, I. S., and Ryshik, I. M.: 'Table of integrals, series and products, 7th Ed.' (New York: Academic Press, 2007).

[24] Hamdi, K. A.: 'A useful lemma for capacity analysis of fading interference channels', IEEE Transactions on Communications, 2010, 58, (2), pp. 411-416.

[25] Abramowitz, M., and Stegun, I. A.: 'Handbook of mathematical functions, with Formulas, Graphs and Mathematical Tables' (New York: Dover Publications, 9th Ed., 1970).

[26] Schlueter, M.: 'MIDACO software performance on interplanetary trajectory benchmarks', Advances in Space Research, 2014, 54, (4), pp. 744-754. 